\title{
Discrepancies between prescribed and defined daily doses: a matter of patients or drug classes?
}

\author{
Thomas Grimmsmann • Wolfgang Himmel
}

Received: 20 May 2010 / Accepted: 5 February 2011 /Published online: 10 March 2011

(C) The Author(s) 2011. This article is published with open access at Springerlink.com

\begin{abstract}
Purpose Defined daily doses (DDD) are used for the measurement of drug utilisation. The aim of the study was to analyse whether differences between DDD and prescribed daily doses (PDD) exist for relevant drug classes such as antihypertensive drugs and, if so, whether they primarily depend on drug classes or patient-related factors. Methods Using the data of a large German statutory health insurance scheme, we analysed continuous prescriptions for the following antihypertensive drug classes: thiazide diuretics, beta-blockers, dihydropyridine calcium channel blockers (CCBs), angiotensin-converting enzyme inhibitors (ACEIs) and angiotensin-II receptor blockers (ARBs). We summed the doses of all dispensed drugs per person during a defined time frame. We calculated the PDD (= total dose divided by the number of days) and expressed them as the PDD:DDD ratio (= amount of DDD per day and person).

Results During the study period, 149,704 patients continuously received an antihypertensive medication. The average PDD:DDD ratio ranged from 0.84 (beta-blockers) to 1.88 (ARBs) and 2.17 (ACEIs). The average prescribed dosage of each drug class remained unchanged, even if the patients had previously received another antihypertensive drug with another PDD:DDD ratio. For example, if patients were switched from a beta-blocker to an ACEI, the PDD:DDD
\end{abstract}

T. Grimmsmann $(\bowtie)$

Medical Review Board of the Statutory Health Insurance Funds

Mecklenburg-Vorpommern,

Schwerin, Germany

e-mail: t.grimmsmann@mdk-mv.de

W. Himmel

Department of General Practice, University of Göttingen,

Göttingen, Germany ratio increased, on average, from 0.79 to 2.17 . Vice versa, the ratio decreased for patients with a drug change from an ACEI to a beta-blocker from 2.06 to 0.75 .

Conclusions Even large differences between DDD and PDD seem to be a matter of drug classes and not primarily of patient characteristics.

Keywords Drug prescriptions · Drug utilisation review . Antihypertensive agents · Pharmacoepidemiology .

Databases

\section{Introduction}

Defined daily doses (DDD) are used as a standard for the measurement of drug utilisation and drug exposure in a population. The WHO [1] defines the DDD as the assumed average maintenance dose per day for a drug used for its main indication in adults.

The DDD does not necessarily reflect the recommended or prescribed daily dose (PDD). Nevertheless, the DDD are widely used for pharmacoepidemiology studies in a setting where a consumption of one DDD per day is implied [2], for example, to compare costs $[3,4]$, to analyse compliance, to calculate disease prevalences $[5,6]$ or to assess the adequacy of a drug supply [7]. Differences between PDD and DDD have been reported in several studies focusing on, for example, antiepileptics, antibacterials, statins or oral hypoglycaemic agents [8-11]. We have ourselves reported rather large differences between PDD and DDD for common drugs such as several angiotensinconverting enzyme inhibitors (ACEIs) or certain antidiabetic drugs [12]. However, these discrepancies may be caused by the severity of diseases or different indications for a drug. 
The aim of the present study was two-fold:

1. To confirm our previous results on a broader database with a focus on a relevant medical indication and not only one class of drugs.

2. To decide whether differences between PDD and DDD are due primarily to patient-related or drug-related factors.

We chose the prescription of antihypertensive drugs, since hypertension is a major risk factor for many cardiovascular and related diseases and high blood pressure has been identified as the leading risk factor for mortality worldwide [13]. Moreover, multiple drugs are recommended for the management of hypertension [14-16] so that the degree of agreement between PDD and DDD should be relevant for pharmacoepidemiology and pharmacoeconomics.

\section{Materials and methods}

\section{Design}

In an observational study, we analysed the prescription data of patients who received antihypertensive drugs continuously ( $>3$ months) from one or several of the five most important drug classes: thiazide diuretics (ATC code C03AA), beta-blockers (C07AB), dihydropyridine calcium channel blockers (CCBs; C08CA), ACEIs (C09AA), or angiotensin-II receptor blockers (ARBs; C09CA).

\section{Database}

The database for the study consisted of prescription data of members insured by a single statutory health insurance (SHI) scheme in the German federal state of MecklenburgWestern Pomerania [12]. This SHI scheme is by far the largest company in this state and insures about one third of the resident population (about 520,000 out of 1.7 million people). We analysed the prescription data between January 2006 and September 2007. For each patient record, the following data were available:

1. The pseudonymised identification number of the insured person.

2. The central pharmaceutical number. This is an identification number providing every detail of the package dispensed, including the ATC classification for the active substance and the information about the number of DDD per package.

3. The date of each prescription.

4. Additional prescription data from October 2005 to December 2007, providing the information as to whether a drug was also prescribed before or after the actual study period (January 2006 to September 2007)

Although the prescriptions do not contain information about the recommended or individual dosage, we tried to find a satisfying approximation for the actual PDD by summing the prescriptions of 3-month intervals only for those patients who also received the analysed drug class both before and after the 3-month period in question. We choose a 3-month time frame because, in general, a patient in Germany with a chronic medication will visit his or her doctor once every 3 months, i.e. from January to March, from April to June etc., to receive a new prescription.

We determined the PDD as the average dispensed daily dose. To calculate the number of DDD for a patient per day from the redeemed prescriptions, i.e. the actual PDD expressed in DDD, we first summed the number of DDD from all packages of all receipts of each patient per 3month period and then divided the summarised prescribed dose by the number of days over these periods of time. This approach, i.e. to calculate the PDD solely from the prescription data, is appropriate since we included only continuous prescriptions and we checked that every patient also received the analysed drug class, both before the study period - as a sort of run-in period to identify prevalent users - and also after the 3-month period under study.

\section{Data analysis}

According to the aims of our study, we compared PDD and DDD (expressed as ratios), with a special focus on drug combinations and drug change, since these events should help us to better discriminate between patient-related and drug-related reasons for differences between DDD and PDD. We defined PDD:DDD ratios, drug combinations and drug change as follows.

\section{PDD:DDD ratio}

We calculated the PDD:DDD ratio, i.e. the PDD denoted in DDD, prescribed for the patient per day and report the average ratio for each of the five drug classes.

\section{Drug combinations}

Then we compared the PDD:DDD ratio for the different drug classes between patients who received only one antihypertensive drug and patients who received a combination of antihypertensive drugs, i.e. drugs from more than one of the analysed drug classes. We did not include fixed combination products in our analysis, because the DDD 
assignment for fixed combinations differs from that for single ingredient products. The DDD for fixed combinations, such as ACEIs or ARBs with diuretics, do not depend on the dose rate of the single ingredients, but are based on the average number of dosing intervals per day. For example, one tablet is the DDD for combinations given once daily, two tablets is the DDD for combinations given twice daily etc. [1]. This method of the DDD assignment would corrupt any attempt to detect discrepancies between PDD and DDD.

\section{Drug change}

In a last step, we compared the PDD:DDD ratios for the different drug classes for those patients whose antihypertensive medication was changed. For this analysis, we selected only patients who received an antihypertensive monotherapy and, again, included only patients who received the antihypertensive drug continuously both before and after the drug change. We defined drug change as a change from one antihypertensive drug class to another class, e.g. from a beta-blocker to an ACEI. The new drug could either start in the same 3-month interval as the former drug ended or in the following 3-month interval (Fig. 1).

\section{Statistics}

Given that the aim of the study was to provide an impression of the magnitude of the differences of prescribed DDD between the different drug classes, descriptive statistics provided by the mean, median and interquartile range (IQR) seemed the most appropriate. No statistical tests were performed as the high number of patients ensured that even small differences between the DDD of different drug classes would be significant, without being clinically relevant.

\section{Results}

During the study period, 149,704 patients continuously received at least one drug from one or more of the five most important antihypertensive drug classes. For three of the five drug classes, the PDD:DDD ratio exceeded 1, i.e. the PDD was higher than the DDD (Table 1); this was most pronounced for ACEIs with a ratio of 2.17 and ARBs with a ratio of 1.88 , on average. Only for beta-blockers was the mean daily dosage less than one DDD. The relevant drugs of each drug class with the respective DDD and PDD:DDD ratios are shown in Table 2. The different substances within each group showed some variability. However, while the PDD:DDD ratios for nearly all beta-blockers did not exceed 1.15 , all ratios were considerably higher for the relevant ACEIs and ARBs.

Interestingly, the upper quartiles of the interquartile range (IQR) for nearly all PDD:DDD ratios were about twice as high as the lower quartiles (Table 2). That is to say that the middle $50 \%$ of the patients were treated with a daily dose between the dose of the lower quartile and twofold this dose. For example, the IQR for bisoprolol ranged from 0.55 to 1.09 , resulting in a PDD between 5 and $10 \mathrm{mg}$ for half of the patients, compared with a DDD of $10 \mathrm{mg}$. In the case of ramipril the IQR ranged from 2.17 to 4.38 , also resulting in a dose between 5 and $10 \mathrm{mg}$ for about half of the patients, this time compared with a DDD of $2.5 \mathrm{mg}$.

Most of the patients (72\%) received only a continuous medication from one of the investigated antihypertensive drug classes, $23 \%$ had two, and the remainder $(5 \%)$ were prescribed drugs from three or more drug classes. If we compare patients who received only one drug and patients who received an antihypertensive treatment composed of more than one drug class, the relation between the PDD: DDD ratios for the different drug classes remained stable.

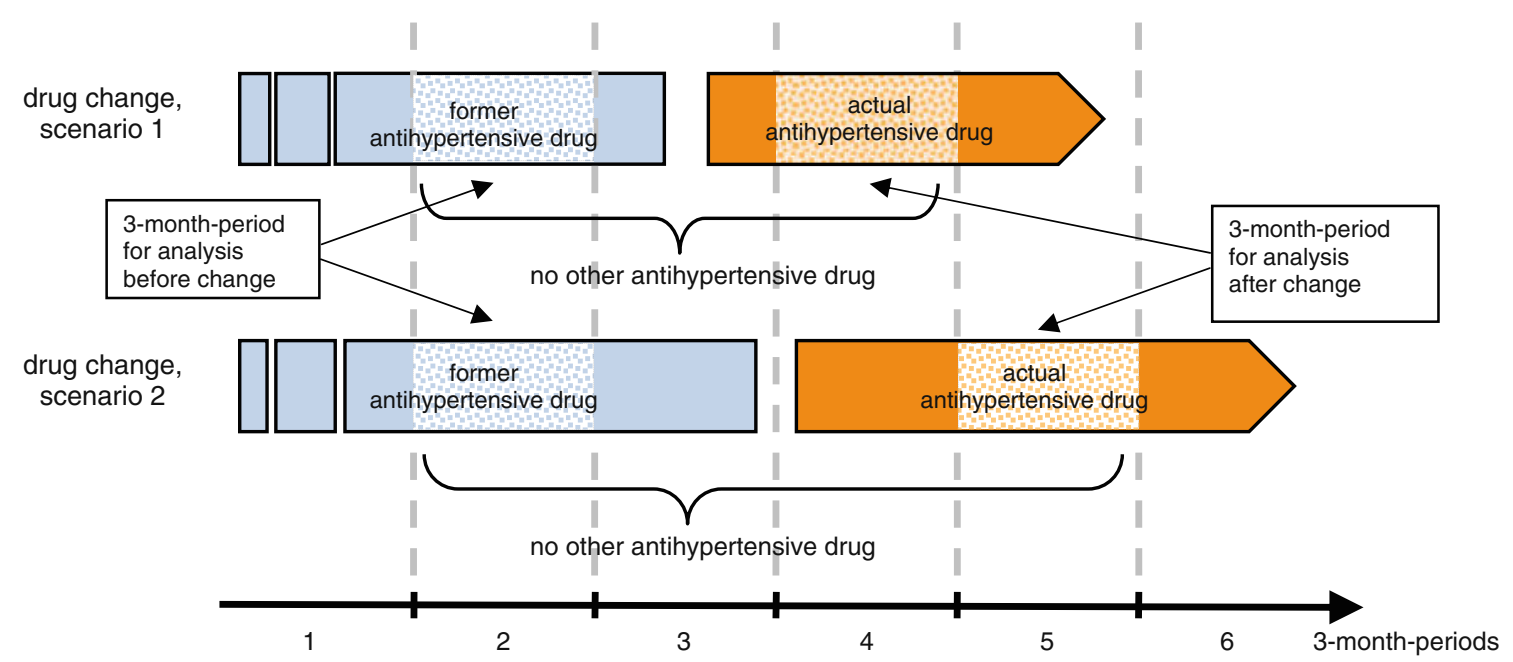

Fig. 1 Two drug change scenarios 
Table 1 The prescribed daily dose $(P D D)$ :defined daily dose $(D D D)$ ratios for different antihypertensive drug classes

${ }^{a}$ Prescribed DDD per patient per day

${ }^{\mathrm{b}}$ Number of patients who received the drug continuously ( $>3$ months)
Drug class (ATC code)

PDD:DDD ratio ${ }^{\mathrm{a}}$

Patients $^{\mathrm{b}}$ Mean (median) $n$

Beta-blockers (C07AB)

$0.84(0.73)$

79,772

Thiazide diuretics (C03AA)

$1.00(1.09)$

8,782

Dihydropyridine calcium channel blockers (C08CA)

$1.51(1.25)$

45,750

Angiotensin-II receptor blockers (C09CA)

$1.88(2.13)$

17,714

ACE inhibitors (C09AA)
Doctors prescribed, for example, ARBs with the second highest PDD:DDD ratio, no matter whether a patient received only one $\mathrm{ARB}$ or additional antihypertensive drugs. The PDD:DDD ratio for all drug classes rose when the number of prescribed antihypertensives increased (Table 3). For example, ARBs were prescribed, on average, with a PDD:DDD ratio of 1.78 in patients who received one antihypertensive drug, compared with 2.22 or 2.35 in

Table 2 The DDD and PDD:DDD ratios of the different substances

\begin{tabular}{|c|c|c|c|c|}
\hline \multirow[t]{2}{*}{ Drug class (ATC code) } & \multirow[t]{2}{*}{ DDD (mg) } & \multirow[t]{2}{*}{ Percentage of patients } & \multicolumn{2}{|c|}{ PDD:DDD ratio } \\
\hline & & & Mean & Median; IQR \\
\hline \multicolumn{2}{|l|}{ Beta-blockers (C07AB) } & 100 & 0.84 & \\
\hline Bisoprolol & 10 & 52 & 0.75 & $0.56 ; 0.55-1.09$ \\
\hline Metoprolol & 150 & 33 & 0.85 & $0.73 ; 0.52-1.15$ \\
\hline Nebivolol & 5 & 7 & 1.15 & $1.10 ; 1.09-1.11$ \\
\hline Talinolol & 100 & 4 & 1.15 & $1.10 ; 0.86-1.37$ \\
\hline Atenolol & 75 & 2 & 0.84 & $0.73 ; 0.37-1.15$ \\
\hline \multicolumn{2}{|c|}{ Other drugs, each with $<2 \%$} & 2 & $1.05-1.23$ & \\
\hline \multicolumn{2}{|c|}{ Thiazide diuretics (C03AA) } & 100 & 1.00 & \\
\hline Hydrochlorothiazide & 25 & 100 & 1.00 & $1.09 ; 0.73-1.10$ \\
\hline \multicolumn{2}{|c|}{ Dihydropyridine calcium channel blockers (C08CA) } & 100 & 1.51 & \\
\hline Amlodipine & 5 & 37 & 1.69 & $1.64 ; 1.10-2.19$ \\
\hline Nitrendipine & 20 & 22 & 1.27 & $1.10 ; 1.00-1.72$ \\
\hline Lercanidipine & 10 & 16 & 1.75 & $1.83 ; 1.10-2.19$ \\
\hline Nifedipine & 30 & 12 & 1.03 & $0.99 ; 0.67-1.36$ \\
\hline Felodipine & 5 & 9 & 1.58 & $1.17 ; 1.10-2.19$ \\
\hline \multicolumn{2}{|c|}{ Other drugs, each with $<2 \%$} & 4 & $0.44-1.65$ & \\
\hline \multicolumn{2}{|c|}{ Angiotensin-II receptor blockers (C09CA) } & 100 & 1.88 & \\
\hline Candesartan & 8 & 26 & 2.50 & $2.15 ; 1.67-3.28$ \\
\hline Valsartan & 80 & 21 & 1.91 & $2.13 ; 1.22-2.15$ \\
\hline Olmesartan medoxomil & 20 & 20 & 1.36 & $1.08 ; 1.07-2.13$ \\
\hline Telmisartan & 40 & 11 & 1.88 & $2.14 ; 1.09-2.15$ \\
\hline Irbesartan & 150 & 11 & 1.76 & $2.13 ; 1.08-2.15$ \\
\hline Losartan & 50 & 7 & 1.45 & $1.09 ; 1.07-2.00$ \\
\hline Eprosartan & 600 & 3 & 1.10 & $1.08 ; 1.07-1.08$ \\
\hline \multicolumn{2}{|l|}{ ACE inhibitors (C09AA) } & 100 & 2.17 & \\
\hline Ramipril & 2.5 & 34 & 3.29 & $3.13 ; 2.17-4.38$ \\
\hline Enalapril & 10 & 33 & 1.70 & $1.47 ; 1.02-2.20$ \\
\hline Lisinopril & 10 & 14 & 1.64 & $1.41 ; 1.09-2.19$ \\
\hline Captopril & 50 & 9 & 1.38 & $1.10 ; 0.56-1.97$ \\
\hline Quinapril & 15 & 5 & 1.20 & $1.10 ; 0.72-1.47$ \\
\hline \multicolumn{2}{|c|}{ Other drugs, each with $<2 \%$} & 5 & $0.93-2.02$ & \\
\hline
\end{tabular}

$\mathrm{IQR}=$ interquartile range $(50 \%$ of the values lie between the lower and upper limits)

Data in bold are drug classes. The rest are substances 
Table 3 The PDD:DDD ratio ${ }^{a}$ for different antihypertensive drug classes used either as monotherapy or in combination (two to five drugs)

\begin{tabular}{lllll}
\hline & \multicolumn{2}{l}{ Number of prescribed antihypertensive drug classes } \\
\cline { 2 - 5 } Drug class (ATC code) & 1 & 2 & 3 & 4 \\
\hline Beta-blockers (C07AB) & 0.82 & 0.88 & 0.94 & 1.01 \\
Thiazide diuretics (C03AA) & 1.00 & 1.00 & 1.01 & 1.02 \\
Dihydropyridine calcium channel blockers (C08CA) & 1.46 & 1.56 & 1.66 & 1.10 \\
Angiotensin-II receptor blockers (C09CA) & 1.78 & 2.01 & 2.22 & 2.39 \\
ACE inhibitors (C09AA) & 2.04 & 2.41 & 2.91 & 3.35 \\
\hline
\end{tabular}

${ }^{\text {a }}$ Prescribed DDD per patient per day

patients taking three or five distinct antihypertensive drug classes respectively.

A continuous antihypertensive monotherapy was changed from one investigated drug class to another in 4,992 patients during the study period. Table 4 shows the PDD:DDD ratios for the initial and subsequent drug. The average dose given of a certain drug class, expressed as the amount of prescribed DDD, was more or less the same regardless of whether or not doctors had changed the prescribed drug class during the study period. For example, 569 patients received an ARB after changing the initial drug class. The average dose for the subsequent ARB ranged within a small interval of between 1.83 and 2.01 DDD (Table 4, "subsequent drug", line C09CA, lower figure), and this interval corresponds to a ratio of 1.88 for all patients receiving an ARB (Table 1). In sharp contrast, the dose of the initial drug that the patient received before the ARB ranged within a large interval of between 0.83 and 2.38 , depending on the initial drug class (Table 4, line C09CA, upper figure).
The same picture arises when we observe the process of drug change from the perspective of the initial drug. If, for example, a patient received a CCB as the initial drug, his or her average dose ranged between 1.43 and 1.65 (Table 4; "initial drug", column "C08CA", upper figure). After drug change, however, the average dose of the subsequent drug ranged within a large interval from 0.84 to 2.3 (Table 4; column "C08CA", lower figure). That is to say, the dosage for a certain drug class prior to (or after) drug change was more or less the same, no matter what drug or dose the patient received after (or prior to) the alteration.

Finally, we analysed drug changes on a substance levelagain only for patients with a continuous monotherapy. We chose switches from bisoprolol to ramipril (242 patients) and vice versa (174 patients). The substances are the most commonly prescribed beta-blockers and ACEIs. The PDD: DDD ratios for bisoprolol in patients before and after a change to ramipril were 0.71 and 0.69 , respectively, while the PDD: DDD ratios for ramipril before and after a change to bisoprolol were 3.16 and 3.12 respectively (data not shown).

Table 4 Prescribed DDD per patient per day for antihypertensive drug classes used as monotherapy in patients with a drug change

\begin{tabular}{|c|c|c|c|c|c|c|}
\hline \multirow[b]{2}{*}{ Subsequent drug } & \multicolumn{6}{|c|}{ Initial drug $^{\mathrm{a}}$} \\
\hline & $\begin{array}{l}\text { C07AB } \\
\text { (mean) }\end{array}$ & $\begin{array}{l}\text { C03AA } \\
\text { (mean) }\end{array}$ & $\begin{array}{l}\text { C08CA } \\
\text { (mean) }\end{array}$ & $\begin{array}{l}\text { C09CA } \\
\text { (mean) }\end{array}$ & $\begin{array}{l}\text { C09AA } \\
\text { (mean) }\end{array}$ & $\begin{array}{l}\text { Patients } \\
(n)\end{array}$ \\
\hline \multirow[t]{2}{*}{ C07AB (Beta-blockers) } & - & 1.01 & 1.48 & 1.83 & 2.06 & 1802 \\
\hline & - & 0.85 & 0.84 & 0.81 & 0.75 & 1614 \\
\hline \multirow[t]{2}{*}{ C03AA (Thiazide diuretics) } & 0.84 & - & 1.65 & 2.19 & 2.17 & 200 \\
\hline & 0.91 & - & 0.97 & 0.98 & 0.96 & 238 \\
\hline \multirow[t]{2}{*}{ C08CA (Calcium channel blockers) } & 0.90 & 1.08 & - & 2.14 & 2.40 & 1099 \\
\hline & 1.52 & 1.70 & - & 1.52 & 1.52 & 1048 \\
\hline \multirow{2}{*}{$\begin{array}{l}\text { C09CA (Angiotensin-II receptor } \\
\text { blockers) }\end{array}$} & 0.83 & 0.98 & 1.54 & - & 2.38 & 473 \\
\hline & 1.90 & 1.84 & 2.01 & - & 1.83 & 569 \\
\hline \multirow[t]{2}{*}{ C09AA (ACE inhibitors) } & 0.79 & 1.09 & 1.43 & 1.69 & - & 1418 \\
\hline & 2.17 & 2.53 & 2.3 & 2.59 & - & 1523 \\
\hline
\end{tabular}

${ }^{a}$ The pairs of figures in the table represent, above, the DDD of the initial drug (i.e. the drug before drug change) and, below, the DDD of the subsequent drug (i.e. the prescribed drug after drug change) 


\section{Discussion}

Summary of main findings

Depending on the drug class, the PDD for the most relevant groups of antihypertensive drugs differed from the DDD; ACEIs were most notable with an average PDD:DDD ratio of 2.17 recorded, followed by the ARBs with a ratio of 1.88. In patients for whom the doctor changed the drug class, the PDD:DDD ratio for the new drug was more or less the same regardless of whether patients had changed to this drug or had always been prescribed this medication. Additionally, the PDD:DDD ratio was also observed to be independent of that of the original drug if they had changed prescription.

Strengths and limitations of the study

One major advantage of this study is the access to and use of a reliable and computer-based data set covering all prescriptions over a sufficient time period. While a single prescription does not contain a dosage recommendation and is not suitable for the calculation of PDD, the large data set in combination with a pseudonymised patient follow-up enabled us to calculate the PDD solely from prescription data. Therefore, we selected only those patients who received continuous medication that started before and ended after our study period, ensuring medication throughout the analysed time frame. Cosentino and colleagues [17] chose a similar study design in their pharmacoepidemiological analysis of drug exposure in a defined population, but unlike this study we only included patients with continuous medication. By doing so, we analysed only prevalent and no incident users. Therefore, we can exclude the fact that a patient with a drug combination in our dataset was exposed to a switch.

Additionally, we had no need to restrict the sample size, which is frequently the case in studies that are based on information collected directly from patients through surveys [18].

Although convinced that our study design characteristics can be expected to ensure a high level of reliability in calculating the respective PDD our results are predicted on prescription data and strictly speaking only on those prescriptions redeemed at pharmacies. Therefore, our only indicator of treatment adherence is that the prescriptions are redeemed.

Comparison with the literature and meaning of the study

This is one of the first studies to compare the PDD and DDD for drug classes prescribed for hypertension as one of the most frequent indications in primary care. The PDDs were higher than the DDDs for ACEIs, ARBs and dihydropyridine CCBs -all three groups belong to the most relevant antihypertensive drugs. Such discrepancies have also been detected in studies on other drugs. For example, Muller and colleagues [9] found a PDD:DDD ratio of 2.97 in amoxicillin-clavulanic acid for the prescription of antibacterials in a university hospital. Consequently, if the calculation were based on this DDD, the number of treatment days in a hospital would be overestimated. In a Czech university hospital study, differences in the PDD:DDD ratio in the prescription of antiepileptics ranged from 0.5 to 4.0 [8]. The PDD for statins was up to twice the DDD in a Norwegian study, which compared statin consumption in different counties [10].

One reason for these discrepancies may be that the drug classes under study, especially ACEIs, could have been prescribed for reasons other than hypertension. This explanation seems at first to be reasonable because ACEIs represent the drugs of choice not only for the treatment of hypertension, but also of heart failure and-in the case of ramipril-cardiovascular prevention, and recommended dosages are usually higher for these indications [19]. For cardiovascular prevention, the recommended dose is $10 \mathrm{mg}$ per day ( $\triangleq 4$ DDD). This seems to be a rather high dose for the treatment of hypertension, but it is still permitted as the maximal dose.

However, according to our analysis of drug change at an individual patient level, we believe we can exclude this potential explanation as being the crucial one. Although we have no information about the indication for the medication of each individual, it is reasonable to assume that the indication for a prescription does not change when the (antihypertensive) drug for the individual patient changes. Predicting that the PDD:DDD ratio depends on patientrelated factors - irrespective of indication or severity of the disease or sociodemographic factors like age or genderthe ratio should not change with a change of the drug. On the contrary, the ratio should change if not patient-related, but drug-related factors are relevant. Our results show clearly that the latter is true. For example, the PDD:DDD ratio for ACEIs was, on average, above 2 for our entire cohort, both for patients before a change to beta-blockers and also for those after change from a beta-blocker. On the other hand, the PDD:DDD ratio was well below 1 for betablockers, irrespective of whether we looked at all patients or only at those before a change to an ACEI or those after a change from an ACEI.

Moreover, we could verify these findings at the level of individual substances for a switch from the ACEI ramipril to the beta-blocker bisoprolol and vice versa. Given the stability of the different PDD:DDD ratios, observed on a patient level after a drug change, it is hard to conceive that these differences are mainly caused by patient-related factors, especially by differences in the indication. 
Other factors, such as the severity of the patient's disease or the doctor's dissatisfaction with the efficacy of a drugno matter if this would lead to a change of drugs or change of dose-also seem to be weak for explaining these consistently observed dosage rates. Again, the abovementioned stability of the different PDD:DDD ratios after drug change is a strong argument against these explanations. Furthermore, differences between the PDD:DDD ratios remain stable independent of the number of different drugs the patients received. Assuming the number of different antihypertensive drugs represents a marker of the severity of the hypertension $[15,16]$, our results show that variations between the PDD:DDD ratios are probably not, or only to a minimal degree, based on differences in the severity of a patient's disease.

Taking this information together, the most logical explanation for the differences between DDD and PDD between the drug classes is that this variability represents a function of the drugs themselves and is not primarily due to factors associated with patients or doctors. We suppose that there are different reasons for differences between prescribed doses and the actual DDDs. One reason might be that the setting and the patient characteristics in clinical studies, which are often the base for the calculation of DDDs, are not equivalent to those observed in primary care settings. Drugs might be highly efficient in clinical studies because of optimal conditions, whereas general practitioners may need to prescribe a higher dose than the DDD in reality. Such "gaps" between pre-market studies and subsequent clinical practice are well-known and sometimes even lead to DDD changes over time [20]. For the ACEIs there is a particular situation insofar as the DDD for all relevant drugs of this group decreased after market introduction. For example, the DDD for ramipril was reduced from 5 to $2.5 \mathrm{mg}$, that for enalapril from 20 to $10 \mathrm{mg}$ respectively $[20,21]$. Without these changes we would have found a far better correspondence between PDD and DDD. Fears regarding the adverse effects of this drug class may have supported a tentative strategy in dose recommendations and led to DDD alteration after market introduction.

\section{Implications and conclusions}

Even for common drug classes such as antihypertensive drugs, it is not possible to calculate the PDD simply from the number of prescribed DDD without the information of the number of patients treated and the time of medication. Although attractive at first glance, DDD do not represent the best means of either analysing the appropriateness of an antihypertensive treatment, or comparing costs between different drug classes, as a result of the drug class-specific discrepancy between PDD and DDD. For the same reasons, it is difficult to assess the degree of drug supply in a population in terms of patients under antihypertensive treatment simply by using the number of prescribed DDD. However, considering these pitfalls adequately, the DDD system is still useful for pharmacoepidemiology.

It may be an interesting issue for future research to study in more detail why the discrepancies between DDD and PDD are rather high in some drug classes and to test our suggestion that these discrepancies may arise from a clinical trial setting that does not necessarily reflect real life or may reflect the manufacturers' as well as prescribers' trust and distrust in the effectiveness and possible adverse effects of new drugs. This could have consequences for the calculation of the DDD for future drugs.

Acknowledgment We are indebted to the AOK MecklenburgVorpommern for permission to perform this study with special thanks to Michael Hewelt for his support.

Conflict of interest The authors declare that they have no conflicts of interest.

Ethics Ethical approval was not necessary due to the nature of the data (secondary data analysis of pseudonymised data).

Open Access This article is distributed under the terms of the Creative Commons Attribution Noncommercial License which permits any noncommercial use, distribution, and reproduction in any medium, provided the original author(s) and source are credited.

\section{References}

1. WHO Collaborating Centre for Drug Statistics Methodology (2009) Guidelines for ATC classification and DDD assignment 2010. http:// www.whocc.no/filearchive/publications/2010guidelines.pdf. Accessed 5 January 2011

2. Merlo J, Wessling A, Melander A (1996) A comparison of dose standard units for drug utilisation studies. Eur J Clin Pharmacol 50:27-30

3. Donohue JM, Fischer MA, Huskamp HA, Weissman JS (2008) Potential savings from an evidence-based consumer-oriented public education campaign on prescription drugs. Health Serv Res 43:1557-1575

4. Schaefer K, Hansen AO, Maerkedahl H, Rehfeld C, Birk HO, Henriksen LO (2007) Changing GPs' prescription patterns through guidelines and feedback. Intervention study. Pharmacoepidemiol Drug Saf 16:695-704

5. Doró P, Benko R, Kosik E, Matuz M, Tóth K, Soós G (2005) Utilization of oral antihyperglycemic drugs over a 7-year period (1998-2004) in a Hungarian population and adherence to drug therapy. Eur J Clin Pharmacol 61:893-897

6. Silwer L, Lundborg CS (2005) Patterns of drug use during a 15 year period: data from a Swedish county, 1988-2002. Pharmacoepidemiol Drug Saf 14:813-820

7. McManus P, Mant A, Birkett D, Hemming M, Lindner J (1999) Examining the adequacy of quantities available for subsidized antidepressant prescriptions in Australia. Pharmacoepidemiol Drug Saf 8:191-195 
8. Koristkova B, Grundmann M, Brozmanova H (2006) Differences between prescribed daily doses and defined daily doses of antiepileptics - therapeutic drug monitoring as a marker of the quality of the treatment. Int J Clin Pharmacol Ther 44:438-442

9. Muller A, Monnet DL, Talon D, Hénon T, Bertrand X (2006) Discrepancies between prescribed daily doses and WHO defined daily doses of antibacterials at a university hospital. Br J Clin Pharmacol 61:585-591

10. Hartz I, Sakshaug S, Furu K, Engeland A, Eggen AE, Njølstad I, Skurtveit S (2007) Aspects of statin prescribing in Norwegian counties with high, average and low statin consumption-an individual-level prescription database study. BMC Clin Pharmacol $7: 14$

11. Duarte-Ramos F, Cabrita J (2006) Using a pharmacoepidemiological approach to estimate diabetes type 2 prevalence in Portugal. Pharmacoepidemiol Drug Saf 15:269-274

12. Grimmsmann T, Himmel W (2010) [Relation between Defined Daily Doses (DDD) and prescribed daily doses: a 3-month analysis of outpatient data from a statutory health insurance company] (in German). Gesundheitswesen 72:412-418

13. Kearney PM, Whelton M, Reynolds K, Muntner P, Whelton PK, He J (2005) Global burden of hypertension: analysis of worldwide data. Lancet 365:217-223

14. Institute for Quality and Efficiency in Health Care (IQWIG) (2009) Different antihypertensive drugs as first line therapy in patients with essential hypertension. Executive Summary of Final Report A05-09, version 1.0 https://www.iqwig.de/download/A0509 Executive Summary Antihypertensive drugs as firstline therapy.pdf. Accessed 5 January 2011

15. Mancia G, De Backer G, Dominiczak A, Cifkova R, Fagard R, Germano G, Grassi G, Heagerty AM, Kjeldsen SE, Laurent S et al (2007) 2007 Guidelines for the management of arterial hyperten- sion: the task force for the management of arterial hypertension of the European Society of Hypertension (ESH) and of the European Society of Cardiology (ESC). J Hypertens 25:1105-1187

16. The National Collaborating Centre for Chronic Conditions (NICE) (2006) Hypertension - management in adults in primary care: pharmacological update. London. http://www.nice.org.uk/nicemedia/ pdf/HypertensionGuide.pdf. Accessed 5 January 2011

17. Cosentino M, Leoni O, Banfi F, Lecchini S, Frigo G (2000) An approach for the estimation of drug prescribing using the defined daily dose methodology and drug dispensation data. Theoretical considerations and practical applications. Eur J Clin Pharmacol $56: 513-517$

18. Harder S, Saal K, Blauth E, Beyer M, Gerlach FM (2009) Appropriateness and surveillance of medication in a cohort of diabetic patients on polypharmacy. Int J Clin Pharmacol Ther 47:104-110

19. European Society of Cardiology, Heart Failure Association of the ESC (HFA), European Society of Intensive Care Medicine (ESICM), Dickstein K, Cohen-Solal A, Filippatos G et al (2008) ESC guidelines for the diagnosis and treatment of acute and chronic heart failure 2008: the Task Force for the diagnosis and treatment of acute and chronic heart failure 2008 of the European Society of Cardiology. Developed in collaboration with the Heart Failure Association of the ESC (HFA) and endorsed by the European Society of Intensive Care Medicine (ESICM). Eur J Heart Fail 10:933-989

20. Heerdink ER, Urquhart J, Leufkens HG (2002) Changes in prescribed drug doses after market introduction. Pharmacoepidemiol Drug Saf 11:447-453

21. WHO Collaborating Centre for Drug Statistics Methodology (2010) DDD alterations from 1982-2010 (last updated: 9 Jun 2010). http://www.whocc.no/atc ddd alterations cumulative / ddd alterations. Accessed 5 January 2011 